\title{
Intermediate landscape disturbance maximizes metapopulation density
}

\author{
Ádám Kun · Beáta Oborny · Ulf Dieckmann
}

Received: 27 January 2008/ Accepted: 16 July 2009

(C) Springer Science+Business Media B.V. 2009

\begin{abstract}
The viability of metapopulations in fragmented landscapes has become a central theme in conservation biology. Landscape fragmentation is increasingly recognized as a dynamical process: in many situations, the quality of local habitats must be expected to undergo continual changes. Here we assess the implications of such recurrent local disturbances for the equilibrium density of metapopulations. Using a spatially explicit lattice model in which the considered metapopulation as well as the underlying landscape pattern change dynamically, we show that equilibrium metapopulation density is maximized at intermediate frequencies of local landscape disturbance. On both sides around this maximum, the metapopulation may go extinct. We show how the position and shape of the intermediate viability maximum is responding to changes in the landscape's overall habitat quality and the population's propensity for local extinction. We interpret our findings in terms of a dual effect of intensified landscape disturbances, which on the one hand
\end{abstract}

Á. Kun $(\bowtie) \cdot$ U. Dieckmann

Evolution and Ecology Program, International Institute for Applied Systems Analysis, Schlossplatz 1,

2361 Laxenburg, Austria

e-mail: kunadam@ludens.elte.hu

\section{Á. Kun · B. Oborny}

Department of Plant Taxonomy and Ecology, Loránd Eötvös University, Pázmány Péter sétány 1/C, 1117 Budapest, Hungary exterminate local populations and on the other hand enhance a metapopulation's capacity for spreading between habitat clusters.

Keywords Environmental heterogeneity · Habitat fragmentation - Percolation . Dynamic landscapes $\cdot$ Cellular automata

\section{Introduction}

Several effects in ecology that were a priori considered detrimental to certain performance measures were later found to be beneficial at intermediate intensity. For example, an intermediate frequency of disturbance has been suggested to maximize species diversity (Grime 1973; Connell 1978). Experimental corroborations of this hypothesis have been reported for rain forests (Molino and Sabatier 2001), phytoplankton communities (Sommer et al. 1993), and experimental microcosms of bacteria (Buckling et al. 2000). Bartha et al. (1997) and Roxburgh et al. (2004) suggested a common theoretical approach to understanding such intermediate maxima. Caswell and Etter (1999) demonstrated, on the basis of a cellular automaton model, that an intermediate frequency of disturbance facilitated the coexistence of competitively superior and fugitive species, while maximizing the equilibrium population size of the inferior species. It has also been shown that an intermediate 
intensity of grazing may maximize plant productivity through the acceleration of a system's nutrient cycling (McNaughton 1979; Hilbert et al. 1985; Dyer et al. 1986; Loreau 1995; de Mazancourt et al. 2001). Furthermore, intermediate disturbance can facilitate cooperation among bacteria in the formation of biofilms (Brockhurst et al. 2007). Intermediate disturbance regimes have thus been demonstrated to possess the potential for maximizing a number of different measures of ecological performance.

The maintenance of species diversity and the optimization of grazing intensity clearly touch on important issues of applied ecology. Another issue of practical importance is the effect of habitat fragmentation on spatially distributed populations (Hanski 1998). Metapopulation models offer widely applied tools for theoretical investigations of habitat fragmentation (Hanski 1998). In particular, spatially explicit metapopulation models, accounting for the two-dimensional structure of many terrestrial habitats, allow for assessing the impact of environmental patchiness on a metapopulation's viability.

Habitat fragmentation is often modelled on the basis of simple random landscapes, termed percolation maps (Gardner et al. 1987; Kun 2007). In these models, a critical transition occurs from a continuous habitat to a fragmented habitat as the overall habitat density is reduced (Gustafson and Parker 1992; Bascompte and Solé 1996). Models based on percolation maps have also proved useful in studies on the effects of habitat heterogeneity on the dynamics of spatially distributed populations (see, e.g., Dytham 1995; Bascompte and Solé 1996; Neuhauser 1998; Hiebler 2000; Hovestadt et al. 2001; Oborny and Kun 2002; Kun and Oborny 2003; Oborny et al. 2007).

Most previous models, however, considered constant landscape patterns. By contrast, highly dynamic landscapes are widespread in nature (Watt 1947; Pickett et al. 2000; Wiens 2000). For example, the dynamics of trees create a dynamic landscape for understorey plants (Verheyen et al. 2004), epiphytes (Snall et al. 2005a, b), and also for forest-dwelling animals (Akçakaya et al. 2004). Succession in patches coupled with local extinctions and disturbance are particularly likely to create changing landscapes (Stelter et al. 1997; Amarasekare and Possingham 2001; Boughton and Malvadkar 2002; Wahlberg et al. 2002; Ellner and Fussmann 2003). Recently, the possibility of turnover in the quality of sites has been incorporated into spatially explicit models of metapopulation dynamics (Keymer et al. 2000). These new models are likely to redefine our understanding of metapopulation persistence, since the destruction and renewal of habitat sites has turned out to be a key element for evaluating such persistence (Keymer et al. 2000; Johst et al. 2002; Wahlberg et al. 2002; Akçakaya et al. 2004; Verheyen et al. 2004; Oborny et al. 2005). It has been demonstrated, in particular, that metapopulation persistence not only depends on the amount of available habitat (Tilman et al. 1994; Bascompte and Solé 1996; Boswell et al. 1998; With and King 1999), but also on the frequency of environmental change (in other words, on the average lifespan of habitat patches; Keymer et al. 2000; Hastings 2003). It is important to note here that in these studies-as well as in ours here-the notion of disturbance refers to changes in the arrangement of habitat patches, whereas other studies have used disturbance in a different sense, as a synonym for habitat destruction.

In our analysis below, a local disturbance makes a habitable patch non-habitable and thus results in the extinction of the local population. We assume that such habitat loss is balanced, on average, by habitat gain through the regeneration or restoration of a patch elsewhere in the landscape, so that the average density of habitable patches remains constant. On this basis, we study the metapopulation dynamics of a species with short-range dispersal in a spatially and temporally heterogeneous, fragmented landscape. We demonstrate for the first time that intermediate levels of local landscape disturbance can maximize metapopulation density, and we try to elucidate the general mechanisms responsible for this phenomenon.

\section{Methods}

A square lattice of $n=300 \times 300$ sites with periodic boundary conditions is used to represent a dynamic landscape. Each site of this landscape is assumed to be either habitable or non-habitable for the population. Habitat sites can be occupied by a local population or they can be empty.

Two processes take place in the system: (a) metapopulation dynamics, changing the occupancy of sites, and (b) landscape dynamics, changing the habitat quality of sites. The latter process creates 
environmental fluctuations through the rearrangement of habitable and non-habitable sites. More specifically, the following two processes are carried out jointly and repeatedly:

(a) First, a site is randomly selected for updating occupancy. If the focal site is occupied, then the local population goes extinct with probability $e$. When extinction occurs, the site becomes empty. If the focal site is empty, it can be (re)colonized from the sites in its von Neumann neighbourhood (four nearest neighbours). (Re)colonization occurs if a randomly picked neighbouring site is occupied. In that case, the focal site becomes occupied.

(b) A second site is then randomly selected for updating habitat quality. If the site is habitable, then its quality is changed to non-habitable with probability $\frac{1}{2} f / p$; if the focal site is non-habitable, then a change to habitable occurs with probability $\frac{1}{2} f /(1-p)$. This transition rule ensures that the fraction of habitable sites in the whole area converges to $P$, while the distribution of habitable sites remains spatially uncorrelated. The rule also means that the frequency at which a site's quality changes, averaged across the whole metapopulation, is $f$. We assume that a site that has just become habitable is initially empty, while a site that has become non-habitable becomes empty.

We confirmed that when sites were not selected independently in the two steps, but instead population and site quality update were conducted on the same site, the qualitative results reported below remained unchanged. In order to assess the generality of our results, we also investigated three alternative scenarios: survival on non-habitable sites, rare long-range dispersal, and synchronous updating. In the first case, a population can survive in a non-habitable site with probability $s \ll 1$. Accordingly, an empty non-habitable site can be colonized with probability $s$ from a randomly chosen occupied neighbouring site, and an occupied habitable site, when becoming non-habitable, can stay occupied with probability $s$. In the second case, a rare long-range dispersal can occur after, on average, each site has been updated once (i.e., after $n$ iterations): a randomly chosen empty habitable site becomes occupied with probability $m$. Another alternative could be to use an exponential dispersal kernel; however it is outside the scope of the current study. In the third case, updating is synchronous, i.e. the occupancy of all sites is updated simultaneously and then the quality of all sites is updated simultaneously.
At the beginning of each simulation, a fraction $p$ of sites is habitable, and all these sites are occupied. The remaining sites are non-habitable and empty. We allow the system to reach equilibrium, by iterating processes (a) and (b) as described above. This takes between 50,000n and 500,000n iterations, and happens faster when $f$ is higher. At equilibrium, the fraction of occupied sites is recorded. For each parameter combination, these fractions are averaged over 11 independent runs. To elucidate how our results may be generalized for the transient dynamics, i.e. before the metapopulation would reaches equilibrium, we also investigated the effects of starting from a fully occupied landscape or from a cluster of $3 \times 3$ occupied sites in an otherwise empty landscape.

\section{Results}

Figure 1 shows how the metapopulation density (the global density or fraction of occupied sites) at equilibrium changes with the frequency $f$ of local landscape disturbance in the special case $e=s=0$, with $p=0.45$. For $e=0$, local populations do not go extinct for any other reason but habitat loss. The figure shows that at low values of $f$, nearly all habitat sites are occupied by the metapopulation: its equilibrium density is close to $p=0.45$. As the frequency of landscape disturbance increases, a growing number of local populations become extinct. Consequently, equilibrium metapopulation density is a strictly decreasing function of disturbance frequency.

When populations may occasionally go extinct even on habitable sites $(e>0)$, an intermediate maximum of equilibrium metapopulation density emerges (Fig. 2). Similarly to Fig. 1, this density drops when the disturbance frequency is increased to large values. Now, however, the density also drops when the disturbance frequency becomes too low.

The position of the intermediate maximum vary both with the global fraction $p$ of habitable sites (Fig. 2 central column) and with the local extinction rate $e$ (Fig. 2 central row The peak's position shifts to lower values of $f$ with the increase of either $e$ or $p$. Notice also that, for $p=0.45$, metapopulation viability is completely lost when the local extinction rate becomes too high (at approximately $e=0.17$ ) and, when the global fraction of habitable sites becomes too low (at approximately $p=0.38$ when $e=0.10$ ). 


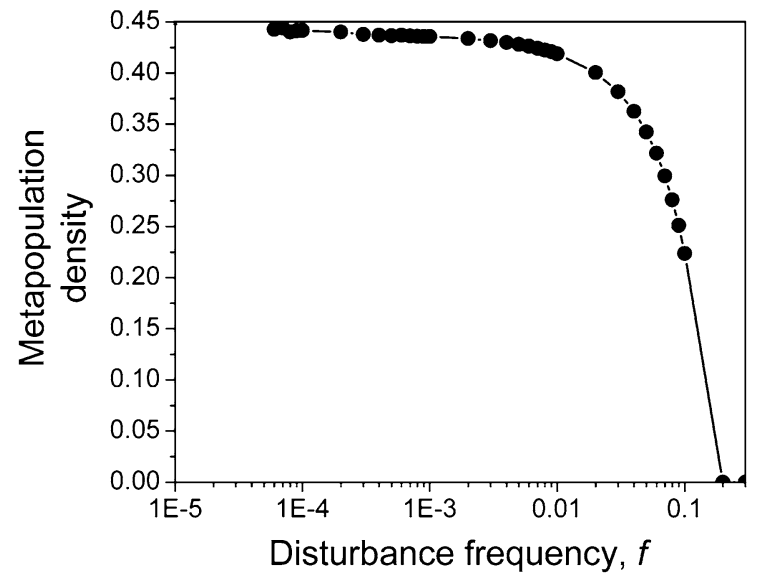

Fig. 1 Equilibrium metapopulation density as a function of the frequency $f$ of landscape disturbance, for an average fraction $p=0.45$ of habitable sites and in the special case $e=0$ (extinction of populations occurs only through habitat loss). Notice that the frequency of landscape disturbance is shown on a logarithmic scale. Each circle represents the average of 11 independent model runs. Coefficients of variation among these model runs were smaller than $1 \%$, and thus error bars are not shown. In each model run, metapopulation density was first equilibrated and then averaged over 1,000 samples spread out in time, with each new sample taken $n$ iterations after the previous one

Even in environments in which the metapopulation can persist, only a fraction of habitable sites are occupied. As expected, the equilibrium metapopulation density increases with habitat quality (central column in Fig. 2) and decreases with extinction rate (central row in Fig. 2).

Underlining the robustness of our results, the intermediate maximum of equilibrium metapopulation density is retained even when populations can survive in non-habitable sites (Fig. 3a), populations exhibit rare long-range dispersal (Fig. 3a), habitat loss only occurs on empty sites (Fig. 3b), or when updating is synchronous (Fig. 3b). By systematically varying all model parameters, we have demonstrated their effects on equilibrium metapopulation density. Despite the absence of a probably impossible analytical treatment, our results thus provide a full analysis of the model's equilibrium behaviour.

Natural systems are not always near equilibrium, instead, they may be subject to large system-scale environmental perturbations, which frequently are anthropogenic. We therefore investigated whether the results can be generalized for transients. We studied the effects of disturbance frequency after starting the metapopulation from extreme initial conditions of maximal and minimal occupation. The former describes a previously undisturbed landscape that becomes exposed to disturbances, while the latter corresponds to a previously empty landscape that becomes invaded by a population. Results are shown in Fig. 4. The intermediate maximum of metapopulation density emerges both after de novo exposure to disturbances and after invasions. In the latter case, the intermediate maximum even occurs right from the onset: this shows that intermediate disturbance frequencies not only maximize equilibrium metapopulation densities but also maximize invasion speed.

\section{Discussion}

Our simple model of metapopulation dynamics unfolding on dynamic landscapes shows that an intermediate frequency of local disturbance maximizes equilibrium metapopulation density and invasion speed. This observation can be explained by two independent effects of landscape disturbance on metapopulation dynamics:

(a) First, a higher frequency of landscape disturbance causes a more rapid extinction of local populations, thus naturally decreasing metapopulation density. At $e=0$, i.e., when disturbance is the only cause of local extinction, this negative effect acts alone, which explains explaining the monotonic decline of metapopulation density with disturbance frequency (Fig. 1). At large disturbance frequencies, this first effect always dominates, explaining, for example, the faster equilibration of a disturbed metapopulation starting from a fully occupied landscape (Fig. 4a).

(b) Second, the rearrangement of habitable sites helps populations to (re)colonize empty habitat patches by creating temporary bridges between habitat clusters (sets of connected habitable sites; Oborny et al. 2007). This bridging effect is clearly visible in Fig. 4b, where it accelerates invasion of a previously empty landscape before effect (a) takes over.

In percolation maps, habitat clusters might be isolated from each other (Gardner et al. 1987). For such maps, with each site being either habitable or non-habitable, it is well known that there exists a critical fraction of habitable sites $\left(p_{c}=0.5923\right.$ for 


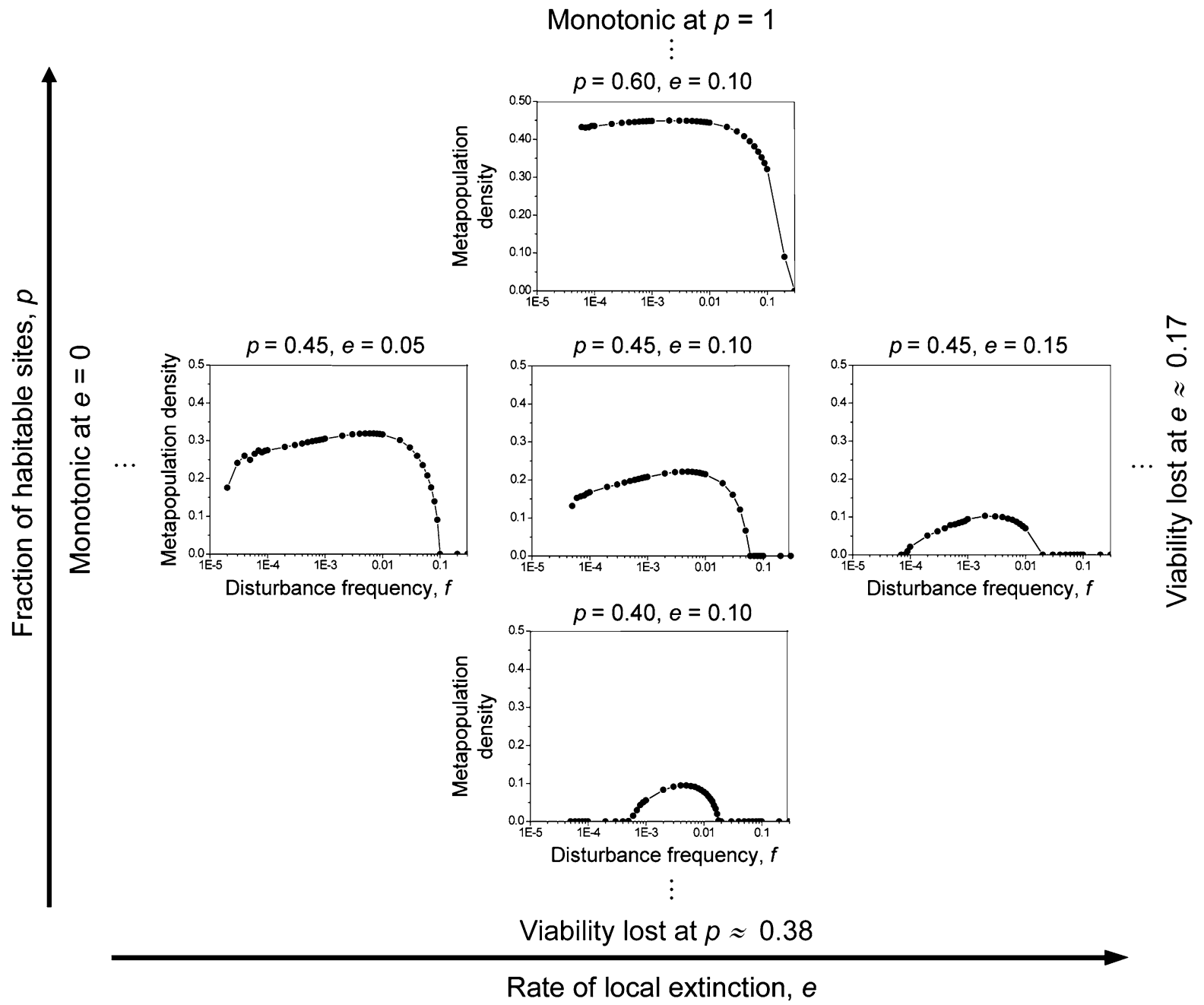

Fig. 2 Equilibrium metapopulation density as a function of the frequency $f$ of landscape disturbance, for $e>0$. Increases in $p$ (from bottom to top) and $e$ (from left to right) show, respectively, the effects of altered fractions of habitable sites

the four-neighbour case), below which the landscape consists of isolated habitat clusters (Stauffer and Aharony 1994). In these settings, the capacity of populations to disperse between habitable sites is seriously limited. The number of isolated habitat clusters is maximal at around $p=0.3$ (Gustafson and Parker 1992; Bascompte and Solé 1996), and is still high at $p=0.45$. Without landscape disturbance, however, most of the isolated habitat clusters remain uncolonized (Fig. 5; see also Oborny and Kun 2002). This implies that, for $p<p_{c}$ and $e>0$, metapopulations go extinct even when the landscape is infinitely large (Oborny et al. 2005). Extensive and of altered rates of local extinction. Notice the existence of an intermediate maximum in each panel (even in the top panel, where $p$ slightly exceeds the percolation threshold). Other details as in Fig. 1

habitat destruction results in a highly fragmented landscape, in which population viability is lost (Bascompte and Solé 1996). Dynamic changes of the landscape introduce the possibility of the emergence of habitable sites ("stepping stones") that connect otherwise isolated habitat clusters. In such a manner, metapopulations may overcome the inherent constraints on spreading imposed by a landscape's structure (Fig. 5). This positive effect of landscape disturbance trades off with the aforementioned negative effect.

Owing to these two competing effects, an optimal frequency of landscape disturbance exists, at which a 

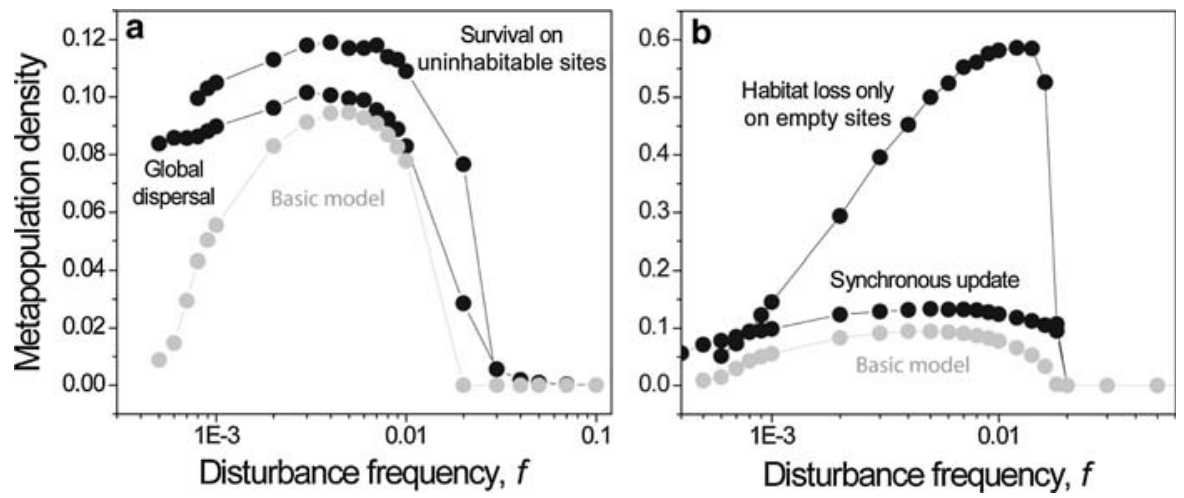

Fig. 3 Equilibrium metapopulation density as a function of the frequency $f$ of landscape disturbance a when populations can survive on non-habitable sites $(p=0.40 ; \quad e=0.1$; $s=0.01)$ and considering rare long-range dispersal $(p=0.40 ; e=0.1 ; m=0.1)$; and $\mathbf{b}$ when habitat loss only

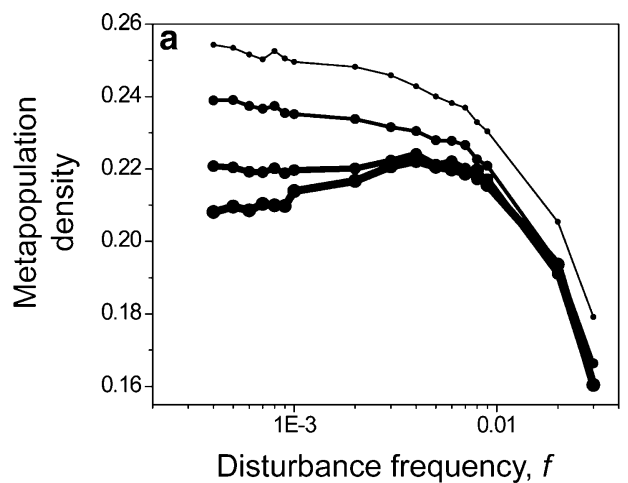

Fig. 4 Metapopulation density at different times during equilibration, as a function of the frequency $f$ of landscape disturbance when the metapopulation is started from a fully occupied landscape and $\mathbf{b}$ from a cluster of $3 \times 3$ occupied sites in an otherwise empty landscape. Metapopulation density is shown after $100 n, 200 n, 500 n$, and 1,000n iterations (thinnest

metapopulation density is maximized (Fig. 2). At higher frequencies of landscape disturbance, the metapopulation may go extinct because it is clobbered by environmental fluctuations, whereas at lower frequencies extinction is due to the scarcity of opportunities leading to the (re)colonization of unoccupied habitat clusters. We demonstrate in the Appendix that the intermediate maximum cannot be captured without accounting for a metapopulation's spatial structure, which shows that this effect crucially depends on spatial correlations among occupied sites. The actual spatial correlation in the system is created by local dispersal, local extinction and the presence of isolated clusters of habitable sites.

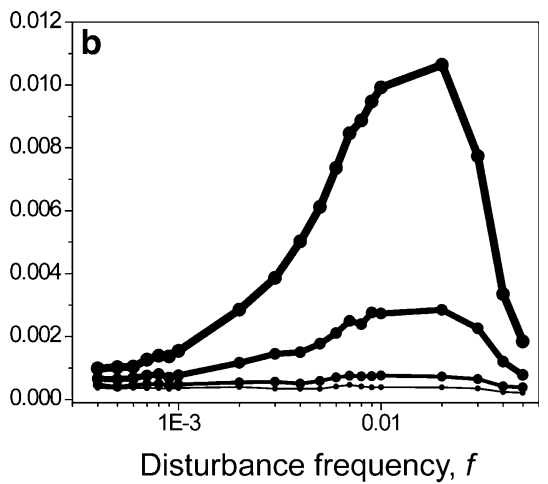

occurs on empty sites or updating is synchronous $(p=0.40$; $e=0.1$ ). The result of the basic model shown in Fig. 2 is repeated here for comparison (grey curve, $p=0.40 ; e=0.1$ ). Other details as in Fig. 1

to thickest curves). Other parameters: $p=0.45$ and $e=0.1$. Other details as in Fig. 1, except that results from each model run were not averaged over time. Coefficients of variation among model runs were smaller than $1 \%$ in a and larger than $1 \%$ in $\mathbf{b}$

The presence of an intermediate maximum of metapopulation density thus requires that local extinctions occur (at least occasionally) due to causes other than habitat loss, and that habitat clusters be sufficiently spatially isolated on the spatial scale of dispersal. The first criterion is usually fulfilled for real-world metapopulations. If habitat sites are more aggregated compared to a random landscape (percolation map), and individual clusters are thus larger but fewer in number, then extinction at the scale of habitat clusters is less frequent, which implies that re-colonization from other clusters is less important.

The second criterion has to be evaluated by examining the spatial characteristics of a landscape 


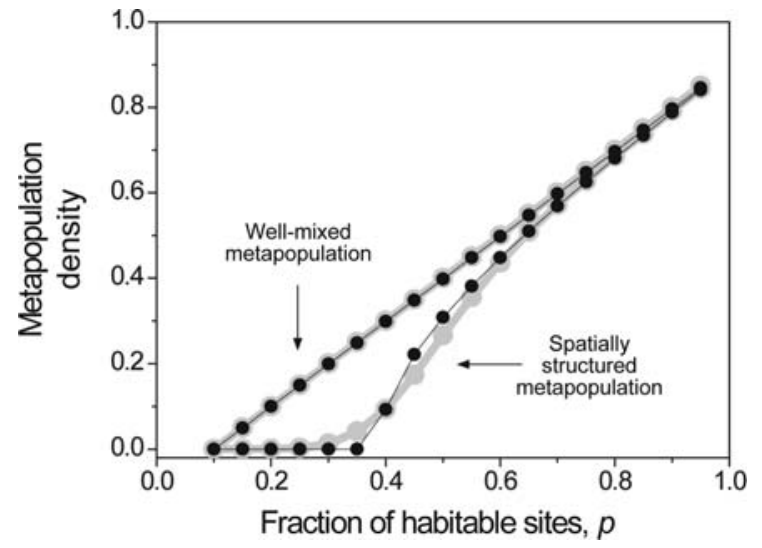

Fig. 5 Equilibrium metapopulation density as a function of the average fraction $P$ of habitable sites, for spatially structured and well-mixed metapopulations without disturbance $(f=0$; grey curves) and with disturbance $(f=0.005$; black curves). Other parameter: $e=0.1$. Other details as in Fig. 1

in conjunction with the ability of a species to "disperse through the matrix" of non-habitable sites. This requires considering three situations: (1) survival on non-habitable sites, (2) long-range dispersal between habitable sites, and (3) sufficiently connected clusters of habitable sites. We discuss these possibilities in turn. (1) In several documented cases, it cannot be excluded that species survive even within that matrix (Andrén 1994; Wiens 2001; Bowne and Bowers 2004). We have shown that when including this possibility in our model, the metapopulation density will continue to show an intermediate maximum, as long as dispersal through the matrix is sufficiently rare compared to the population's rate of spread across habitable sites (Fig. 3a). (2) Rare longrange dispersal and subsequent establishment of populations is another mechanism by which the spatial isolation of habitat clusters is alleviated. While rare in many systems, long-range dispersal is more common in others (Wiens 2000). It is therefore important that our general result is robust to rare dispersal events through which individuals are bypassing the intervening inhospitable matrix, for example by seed dispersal (Fig. 3b). While a population with long-range dispersal capabilities can colonise habitats vacated by extinction, it will still benefit from intermediate levels of disturbance if the establishment of dispersing individuals is sufficiently rare. Establishment from seeds can indeed be very low in populations of clonal plants, for which the main mode of spread is vegetative growth (Eriksson
1997). (3) If the habitat is not much fragmented, populations can percolate through one large cluster of habitable sites. In such a situation, the bridging effect of disturbances is not very important, as it only connects smaller unconnected clusters to the already existing large cluster that greatly facilitates a population's spread. It is important to point out, however, that the intermediate maximum still occurs, if only weakly, even when the fraction of habitable sites is raised above a landscape's percolation threshold, as illustrated by the top panel in Fig. 2 .

Several studies have indicated the importance of modest disturbance for the maintenance of natural populations. For example, the population of a grasshopper species (Bryodema tuberculata) on gravel bars along braided rivers in the Northern Alps depends on irregular floods creating open areas that would otherwise be overgrown and become nonhabitable (Stelter et al. 1997). In another flood plain, habitat ice scour disturbs local populations of lousewort (Pedicularis furbishiae), but the pruning effect of the ice drift also keeps the vegetation sparse, which is essential for the growth of this species (Menges 1990). As yet another example, localized fires can remove plant cover and thus create new habitat, for example, for checkerspot butterflies (Euphydrias gilettii; Debinski 1994) and lichen grasshoppers (Trimerotopis saxatilis; Gerber and Templeton 1996). Describing a different but similar phenomenon, Nee and May (1992) showed that if a competitively inferior species can coexist with a dominant competitor, its equilibrium density exhibits a maximum at intermediate habitat density. It could be interesting to extend of our model to metapopulations in which available habitat is limited not only by a fragmented landscape of potential habitat, but also by the presence of a strong competitor.

Our model predicts that the management of disturbances, or of restoration efforts counterbalancing habitat loss, can increase a metapopulation's density when landscapes are fragmented, survival on non-habitable sites is low, local extinction risks are not extreme, and dispersal is mostly short-range. In general, the negative effects of habitat fragmentation will often be difficult to counteract (Fahrig 1998, 2003; Harrison and Bruna 1999; Falther and Bevers 2002) and are bound to differ much among species and landscapes (Fahrig 2001). However, when countermeasures can be taken in a (cost-)effective manner, 
our results offer two relevant insights. First, we have shown how sensitively a metapopulation's equilibrium density can depend on the frequency of landscape disturbances, as illustrated by the steep parts of curves in Fig. 2. This sensitivity, and therefore the benefit-to-cost ratio of appropriate management measures, is highest near the maximum disturbance frequency that a metapopulation can sustain. For natural metapopulations threatened by extinction, this realization is especially salient. Second, our results have highlighted an unexpected beneficial effect of recurrent destruction and creation of local habitats, resulting in a minimum disturbance frequency that a metapopulation can sustain. In the vicinity of that lower threshold, small changes in disturbance frequency again go a long way in elevating metapopulation density. What is perhaps even more important is that our findings also show how well-meant attempts to improve a metapopulation's viability by reducing the frequency of disturbances can backfire, if they are applied on the wrong side of the intermediate maximum.

Observations of systems in which landscape dynamics play an essential role in population dynamics have lead to a re-evaluation of the role of disturbance and of the conditions for (meta)population persistence. Our results confirm that the dreaded effect of the percolation threshold (Gardner et al. 1987; Stauffer and Aharony 1994; Bascompte and Solé 1996; Boswell et al. 1998) might be alleviated on dynamic landscapes (see also Keymer et al. 2000; Roy et al. 2004; Oborny et al. 2007) through the occasional formation of bridges between habitat clusters. Accordingly, landscape disturbance can increase metapopulation density, resulting in increased persistence. These findings may enable innovative strategies for landscape management. In particular, when only a limited number of sites can be protected, our results highlight the importance of focusing efforts on creating habitable sites at new locations, so as to allow the escape of populations from existing enclosures. Under some conditions, a moderate amount of dynamic changes in the pattern of habitable sites suffices to significantly increase a metapopulation's viability.

Acknowledgments We are grateful to Géza Meszéna, Éva Kisdi, György Szabó, and Hans Metz for valuable and enjoyable discussions about this project, and to Péter Mandl for helpful comments on an earlier draft of this manuscript. The project was subsidized by the Hungarian Scientific
Research Fund (OTKA K61534), the Hungarian Ministry of Education (FKFP 0187/1990, István Széchenyi Scholarship), and the Hungarian Academy of Sciences (János Bolyai Scholarship). A.K. acknowledges financial support from the European Science Foundation's Theoretical Biology of Adaptation Programme, enabling participation in IIASA's Young Scientists Summer Program, as well as subsequent visits to IIASA. Postdoctoral fellowships of Á.K. were funded by OTKA (D048406) and through a Lise Meitner grant to Á.K. and U.D. by the Austrian Science Fund (M983-N18). B.O. acknowledges support from the International Program of the Santa Fe Institute, USA. U.D. acknowledges financial support by the European Science Foundation, the Austrian Science Fund, the Austrian Federal Ministry of Education, Science, and Cultural Affairs, the Vienna Science and Technology Fund, and the European Research Training Network ModLife (Modern Life-History Theory and its Application to the Management of Natural Resources), funded through the Human Potential Programme of the European Commission.

\section{Appendix: Well-mixed metapopulations}

When long-range dispersal is much more frequent than short-range dispersal, the metapopulation described by our model is well-mixed, so that all spatial correlations in the occupancy of sites are lost. This enables a simple analytical treatment, which we include here so as to demonstrate the crucial importance of spatial structure for our results.

Assuming a well-mixed metapopulation with a large number $n$ of sites, the dynamics of the proportion of habitable occupied sites, $N$, is given by

$\frac{d N}{d t}=N(p-N)-N\left(e+\frac{1}{2} f / p\right)$

where time $t$ is measured in units of $n$. This is a special case of the mean-field metapopulation dynamics studied by Keymer et al. 2000; in our model, the fraction $p$ of habitable sites and the total number of sites remain constant).

The equilibrium metapopulation density $N^{*}=$ $\max \left(0, p-e-\frac{1}{2} f / p\right)$ decreases as $f$ increases. This shows that, as expected, mean-field models cannot capture the bridging effect of landscape disturbance and therefore only account for the local extinctions caused by such disturbance. Moreover, the effect of a small disturbance frequency $f$ on $N^{*}$ is negligible for a well-mixed metapopulation, whereas it leads to marked changes in the equilibrium metapopulation densities of a spatially structured metapopulation (Fig. 5). 


\section{References}

Akçakaya HR, Radeloff VC, Mladenoff DJ, He HS (2004) Integrating landscape and metapopulation modeling approaches: viability of the sharp-tailed grouse in a dynamic landscape. Conserv Biol 18:526-537

Amarasekare P, Possingham H (2001) Patch dynamics and metapopulation theory: the case of successional species. J Theor Biol 209:333-344

Andrén H (1994) Effect of habitat fragmentation on birds and mammals in landscapes with different proportion of suitable habitat: a review. Oikos 71:355-366

Bartha S, Czárán T, Scheuring I (1997) Spatiotemporal scales of non-equilibrium community dynamics: a methodological challenge. N Z J Ecol 21:199-206

Bascompte J, Solé RV (1996) Habitat fragmentation and extinction threshold in spatially explicit models. J Anim Ecol 65:465-473

Boswell GP, Britton NF, Franks NR (1998) Habitat fragmentation, percolation theory and the conservation of a keystone species. Proc R Soc Lond B 265:1921-1925

Boughton D, Malvadkar U (2002) Extinction risk in successional landscapes subject to catastrophic disturbances. Conserv Biol 6: article no. 2

Bowne DR, Bowers MA (2004) Interpatch movements in spatially structured populations: a literature review. Landscape Ecol 19:1-20

Brockhurst MA, Buckling A, Gardner A (2007) Cooperation peaks at intermediate disturbance. Curr Biol 17:761-765

Buckling A, Kassen R, Bell G, Rainey PB (2000) Disturbance and diversity in experimental microcosms. Nature 408:961-964

Caswell H, Etter R (1999) Cellular automaton models for competition in patchy environments: facilitation, inhibition, and tolerance. Bull Math Biol 61:625-649

Connell JH (1978) Diversity in tropical rain forest and coral reefs. Science 199:1302-1310

de Mazancourt C, Loreau M, Dieckmann U (2001) Can the evolution of plant defense lead to plant-herbivore mutualism? Am Nat 158:109-123

Debinski DM (1994) Genetic diversity assessment in a metapopulation of the butterfly Euphydryas gillettii. Biol Conserv 70:25-31

Dyer M, DeAngelis DL, Post WM (1986) A model of herbivore feedback on plant productivity. Math Biosci 79: $171-184$

Dytham C (1995) The effect of habitat destruction pattern on species persistence: a cellular model. Oikos 74:340-344

Ellner SP, Fussmann G (2003) Effects of successional dynamics on metapopulation persistence. Ecology 84:882-889

Eriksson O (1997) Clonal life histories and the evolution of seed recruitment. In: van Groenendael J, de Kroon H (eds) The ecology and evolution of clonal plants. Backhuys, Leiden, pp 211-226

Fahrig L (1998) When does fragmentation of breeding habitat affect population survival? Ecol Model 105:273-292

Fahrig L (2001) How much habitat is enough? Biol Conserv 100:65-74
Fahrig L (2003) Effects of habitat fragmentation on biodiversity. Ann Rev Ecol Evol Syst 34:487-515

Falther C, Bevers M (2002) Patchy reaction-diffusion and population abundance: the relative importance of habitat amount and arrangement. Am Nat 159:40-56

Gardner RH, Milne BT, Turner MG, O'Neill RV (1987) Neutral models for the analysis of broad-scale landscape pattern. Landscape Ecol 1:19-28

Gerber AS, Templeton AR (1996) Population sizes and withindeme movement of Trimerotropis saxatilis (Acrididae), a grasshopper with a fragmented distribution. Oecologia 105:343-350

Grime JP (1973) Competitive exclusion in herbaceous vegetation. Nature 242:344-347

Gustafson EJ, Parker GR (1992) Relationships between landcover proportions and indices of landscape spatial pattern. Landscape Ecol 7:101-110

Hanski I (1998) Metapopulation dynamics. Nature 396:41-49

Harrison S, Bruna E (1999) Habitat fragmentation and largescale conservation: what do we know for sure? Ecography 22:225-232

Hastings A (2003) Metapopulation persistence with agedependent disturbance or succession. Science 301: $1525-1526$

Hiebler D (2000) Populations on fragmented landscapes with spatially structured heterogeneities: landscape generation and local dispersal. Ecology 81:1629-1641

Hilbert DW, Swift DM, Detling JK, Dyer M (1985) Relative growth rates and the grazing optimization hypothesis. Oecologia 51:14-18

Hovestadt T, Messer S, Poethke HJ (2001) Evolution of reduced dispersal mortality and 'fat-tailed' dispersal kernels in autocorrelated landscapes. Proc R Soc Lond B 268:385-391

Johst K, Brandl R, Eber S (2002) Metapopulation persistence in dynamic landscapes: the role of dispersal distance. Oikos 98:263-270

Keymer JE, Marquet PA, Velasco-Hernández JX, Levin SA (2000) Extinction threshold and metapopulation persistence in dynamic landscapes. Am Nat 156:478-494

Kun Á (2007) Generation of temporally and spatially heterogeneous landscapes for models of population dynamics. Appl Ecol Environ Res 4:73-84

Kun Á, Oborny B (2003) Survival and competition of clonal plant populations in spatially and temporally heterogeneous habitats. Community Ecology 4:1-20

Loreau M (1995) Consumers as maximisers of matter and energy flow in ecosystems. Am Nat 145:22-42

McNaughton SJ (1979) Grazing as an optimization process: grass-ungulate relationship in the Serengeti. Am Nat 113:691-703

Menges E (1990) Population viability analysis for an endangered plant. Conserv Biol 4:52-61

Molino J, Sabatier D (2001) Tree diversity in tropical rain forests: a validation of the intermediate disturbance hypothesis. Science 294:1702-1704

Nee S, May RM (1992) Dynamics of metapopulations: habitat destruction and competitive coexistence. Anim Behav 61:37-40 
Neuhauser C (1998) Habitat destruction and competitive coexistence in spatially explicit models with local interaction. J Theor Biol 193:445-463

Oborny B, Kun Á (2002) Fragmentation of clones: how does it influence dispersal and competitive ability? Evol Ecol 15:319-346

Oborny B, Meszéna G, Szabó G (2005) Dynamics of populations on the verge of extinction. Oikos 109:291-296

Oborny B, Szabó G, Meszéna G (2007) Survival of species in patchy landscapes: percolation in space and time. In: Storch D, Marquet PA, Brown JH (eds) Scaling biodiversity. Cambridge University Press, Cambridge

Pickett STA, Cadessano ML, Jones CG (2000) Generation of heterogeneity by organisms: creation, maintenance and transformation. In: Hutchings MJ, John E, Stewart AJ (eds) The ecological consequences of environmental heterogeneity. Blackwell, Oxford, pp 33-52

Roxburgh SH, Shea K, Wilson JB (2004) The intermediate disturbance hypothesis: patch dynamics and mechanisms of species coexistence. Ecology 85:359-371

Roy M, Pascual M, Levin SA (2004) Competitive coexistence in a dynamical landscape. Theor Popul Biol 66:341-353

Snall T, Ehrlen J, Rydin H (2005a) Colonization-extinction dynamics of an epiphyte metapopulation in a dynamic landscape. Ecology 86:106-115

Snall T, Pennanen J, Kivisto L, Hanski I (2005b) Modelling epiphyte metapopulation dynamics in a dynamic forest landscape. Oikos 109:209-222

Sommer U, Padisák J, Reynolds CS, Juhász-Nagy P (1993) Hutchinson's heritage: the diversity-disturbance relationship in phytoplankton. Hydrobiologia 249:1-7
Stauffer D, Aharony A (1994) Introduction to percolation theory. Taylor and Francis, London Revised second edition

Stelter C, Reich M, Grimm V, Wissel C (1997) Modelling persistence in dynamic landscapes: lessons from a metapopulation of grasshopper Bryodema tuberculata. J Anim Ecol 66:508-518

Tilman D, May RM, Lehman CL, Nowak MA (1994) Habitat destruction and the extinction debt. Nature 371:65-66

Verheyen K, Vellend M, Van Calster H, Peterken G, Hermy M (2004) Metapopulation dynamics in changing landscapes: a new spatially realistic model for forest plants. Ecology 85:3302-3312

Wahlberg N, Klemetti T, Hanski I (2002) Dynamic populations in a dynamic landscape: the metapopulation structure of the marsh fritillary butterfly. Ecography 25:224-232

Watt AS (1947) Pattern and process in the plant community. J Ecol 35:1-22

Wiens JA (2000) Ecological heterogeneity: an ontogeny of concepts and approaches. In: Hutchings MJ, John E, Stewart AJ (eds) The ecological consequences of environmental heterogeneity. Blackwell, Oxford, pp 9-31

Wiens JA (2001) The landscape context of dispersal. In: Clobert J, Danchin E, Dhondt AA, Nichols JD (eds) Dispersal. Oxford University Press, Oxford, pp 96-109

With KA, King AW (1999) Extinction thresholds for species in fractal landscapes. Conserv Biol 13:314-326 